\title{
Measuring the Impacts of Teachers: Reply to Rothstein
}

\author{
Raj Chetty, John Friedman, and Jonah Rockoff
}

\section{Online Appendix A. Teacher Followers and Prior Scores}

In this appendix, we provide further detail on why including teachers who follow students across grades can produce correlations between changes in current VA and prior test scores across cohorts.

Consider the relationship between changes in math test scores and math teacher VA from 1994 to 1995 in 5th grade in a given school. Suppose a teacher with high estimated VA leaves 5th grade after 1994 and is replaced by a teacher with average VA; assume there are no other changes in the teaching roster. We know that the high-VA teacher who departed did not teach the children who were in grade 5 in 1995 when they were in 4th grade in 1994 (because she taught 5th grade in 1994). However, she may have taught the children who were in grade 5 in 1994 when they were in 4th grade in 1993. As a result, the high VA of the departing teacher is positively correlated with lagged test scores of the cohort that reaches 5 th grade in 1994, but not the test scores of those who reach 5th grade in 1995. This effect makes lagged (4th grade) test scores fall on average across the two cohorts. Since (by construction) teacher VA is also falling in this example, there is a positive correlation between changes in lagged (4th grade) scores across the two cohorts and mean teacher VA.

It is useful to distinguish between two separate channels that drive this correlation. The first channel is fluctuations in student test scores that are not related to the persistent component of teacher value-added, i.e., noise in student test scores. The teachers in 5th grade in 1994 could have higher estimated VA simply because her students in 4th grade test in 1993 did particularly well by chance (e.g., because the curriculum in the school happened to be well aligned with the test questions that year). This creates a mechanical correlation between lagged scores and VA estimates but has no bearing on our estimate of forecast bias using current test scores. Second, the correlation could be driven by teacher treatment effects. If the 5th grade teachers in 1994 were of truly high quality, they would affect the performance of 4th graders in 1993 (because some of them taught 4th grade in 1993), but not the 4th graders in 1994 (because we know they are teaching 5th grade in 1994). Note that, in contrast to the first channel, the direct treatment effect of teachers in prior grades could potentially bias our estimate of $\lambda$, as having better teachers in prior school years can 
increase current scores. The magnitude of bias depends upon the rate of fade-out in the sample where a teacher teaches the same child twice. The fact that the estimates of $\lambda$ do not change when we exclude followers (Columns 1 and 2 of Table 4) shows that in practice, there is little or no bias in our estimate of $\lambda$ from this latter channel.

\section{Online Appendix B. Residualization Using Within-Teacher Variation}

In a standard partial-regression implementation of a multivariate regression model, one must residualize both the left- and right-hand side variables with respect to the covariates to obtain a consistent estimate of the regression coefficient of interest. In this appendix, we show why one should not residualize the right hand size variable (teacher VA) with respect to covariates $X_{i t}$ when the effects of the covariates on long-term outcomes $Y_{i t}^{*}$ are estimated using within-teacher variation.

Suppose the statistical model for earnings is

$$
\left.Y_{i}^{*}=\alpha+\kappa m_{j}+\beta^{Y} X_{i t}+\right\} \eta_{i t}
$$

with $\eta_{i t}$ orthogonal to $X_{i t}$ and $m_{j}$ defined as normalized teacher VA, as in equations (2) and (4) in CFR-II.

First, observe that if we knew $\beta^{Y}$, we could mechanically construct $Y_{i t}=Y_{i}^{*}-\beta^{Y} X_{i t}$ and then simply regress $Y_{i t}$ on $m_{j}$ (without including any controls) to obtain an unbiased estimate of $\kappa$ under the selection on observables assumption in CFR-II (Assumption 2). In this case, residualizing value-added $m_{j}$ with respect to $X_{i t}$ would not yield a consistent estimate of $\kappa$ because the true model is $Y_{i t}=\alpha+\kappa m_{j}+\eta_{i t}$.

Now suppose that we do not know $\beta^{Y}$ and estimate it using an OLS regression (without teacher fixed effects) of the form

$$
Y_{i}^{*}=a+b^{Y} X_{i t}+\varepsilon_{i t}
$$

Here $b^{Y}$ does not provide a consistent estimate of $\beta^{Y}$ if teacher VA $m_{j}$ is correlated with $X_{i t}$ : $b^{Y}$ converges to $\beta^{Y}+\kappa \operatorname{Cov}\left(m_{j}, X_{i t}\right) / \operatorname{Var}\left(X_{i t}\right)$. Since $b^{Y}$ is not a consistent estimate of $\beta^{Y}$, one cannot simply regress $Y_{i}^{*}-b^{Y} X_{i t}$ on $m_{j}$ to obtain a consistent estimate of $\kappa$. Intuitively, the reason one must residualize both $Y_{i t}$ and $m_{j}$ in a multivariate regression is that an OLS regression of $Y_{i t}$ on $X_{i t}$ does not produce a consistent estimate of the structural parameter $\beta^{Y}$ in (1) because it partly picks up the effect of $m_{j}$, which is correlated with $X_{i t}$. To correct for the incorrect estimate of $\beta^{Y}$, one must residualize the right-hand-side variable $m_{j}$ with respect to $X_{i t}$ and then regress the earnings residuals $Y_{i t}$ on the VA residuals $\tilde{m}_{j t}=m_{j}-\gamma X_{i t}$. 
Now consider our approach, where we estimate $\beta^{Y}$ using an OLS regression with teacher fixed effects $a_{j}$ :

$$
Y_{i}^{*}=a_{j}+b_{f}^{Y} X_{i t}+\varepsilon_{i t}^{\prime}
$$

Here, the coefficient $b_{f}^{Y}$ is identified purely from within-teacher variation in $X_{i t}$ that is mechanically uncorrelated with variation in $m_{j}$. Therefore, under the model in (1), the coefficient $b_{f}^{Y}$ converges to $\beta^{Y}$ and hence regressing $Y_{i}^{*}-b_{f}^{Y} X_{i t}$ on $m_{j}$ yields a consistent estimate of $\kappa$, for the same reason that regressing $Y_{i}^{*}-\beta^{Y} X_{i t}$ on $m_{j}$ when $\beta^{Y}$ is known yields a consistent estimate of $\kappa$ in the first case considered above. In contrast, using residual VA $\tilde{m}_{j t}=m_{j}-\gamma X_{i t}$ in the second regression would yield an inconsistent estimate of $\kappa$. Intuitively, when we use within-teacher variation to estimate $\beta^{Y}$, we immediately obtain a consistent estimate of the effect of $X$ on earnings that is not contaminated by the correlation with teacher value-added. Hence, one simply has to regress the outcome residual on VA to estimate the effect of teacher VA in the second stage. 


\section{Online Appendix C. Stata Code for Simulations}

\section{Imputation of Missing Data}

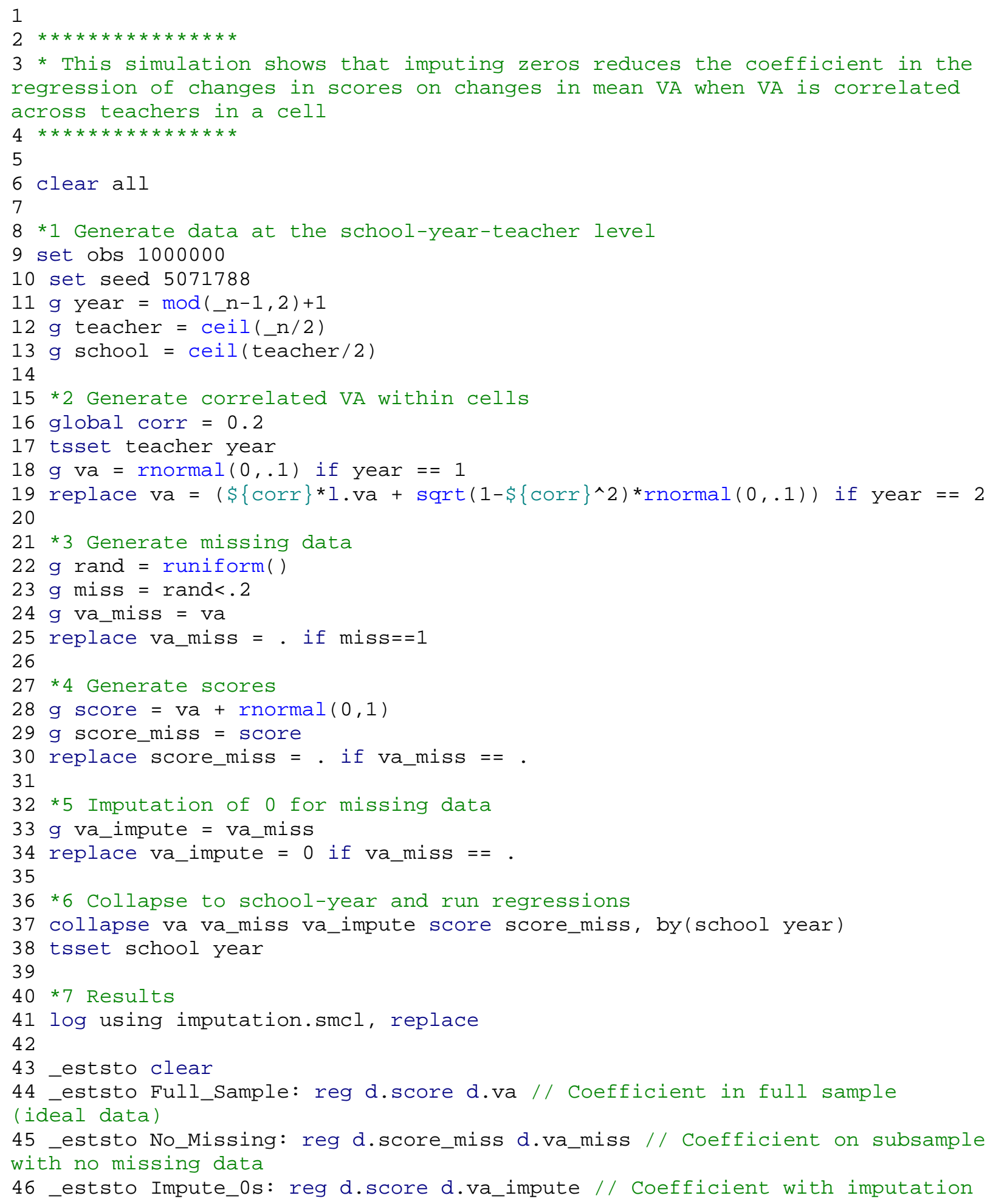


is downward-biased

49 esttab_all, mtitles title("Missing Data Imputation Simulation Results")

se not

$50 \log$ close

\section{Prior Test Scores (Table 3)}

1 *This simulation shows that prior test score changes with be correlated with changes in current mean VA across cohorts when there are school-year level shocks

2 *Simulates data for one subject, so shocks should be interpreted as schoolyear-subject shocks

3 *The program simulates class-level data, incorporating teacher effects, class effects, student-level noise, and a school-year shock common to all classrooms.

4

5 clear all

6 set seed 717806

7 set more off

8

9 * Parameters governing simulation

10 global min_grade $=3 / /$ Minimum grade level (for readability)

11 global min_year = $1992 / /$ Start year (for readability)

12 global n_school $=10000 / /$ Number of schools

13 global n_year $=6 / /$ Number of years

14 global n_grade $=6 / /$ Number of grades taught per school

15 global n_rooms $=4 / /$ Number of classrooms per school and grade

16 global n_class $=25 / /$ Number of students per class

17 global var_tot $=0.25 / /$ Total variance of scores

18 global sd_va $=0.10 / /$ Standard deviation of value added

19 global sd_class_shock $=0.08 / /$ Standard deviation of classroom-level shocks

20 global sd_sy_shock $=.08 / /$ Standard deviation of school-by-year shocks

21 global rho_sy $=0.35 / /$ Autocorrelation on school-by-year shocks

22

23 * Generate basic data

24 set obs $=\$\left\{\mathrm{n} \_\right.$school $\} * \$\left\{\mathrm{n} \_\right.$grade $\} * \$\left\{\mathrm{n} \_\right.$rooms $\}$* $\$\left\{\mathrm{n} \_\right.$year $\}$'

$25 \mathrm{~g}$ school $=\operatorname{ceil}\left(\_\mathrm{n} /\left(\$\left\{\mathrm{n} \_\right.\right.\right.$grade $\} * \$\left\{\mathrm{n} \_\right.$rooms $\} * \$\left\{\mathrm{n} \_\right.$year $\left.\left.\}\right)\right)$

$26 \mathrm{~g}$ grade $=\bmod \left(\operatorname{ceil}\left(\_\mathrm{n} /\left(\$\left\{\mathrm{n} \_\right.\right.\right.\right.$rooms $\} * \$\left\{\mathrm{n} \_\right.$year $\left.\left.\}\right)\right)-1, \$\left\{\mathrm{n} \_\right.$grade $\left.\}\right)+$ $\$\{$ min_grade $\}$

27 g teacher $=\operatorname{ceil}\left(\_\mathrm{n} / \$\left\{\mathrm{n} \_\right.\right.$year $\left.\}\right)$

$28 \mathrm{~g}$ year $=\bmod \left(\mathrm{n}-1, \$\left\{\mathrm{n} \_\right.\right.$year $\left.\}\right)+\$\{$ min_year $\}$

$29 \mathrm{~g}$ id $=\operatorname{rnormal}()$

30

31 * Replace some teachers in 1997

32 * Only in grades 5-8 (since others not used in experiment at end)

$33 \mathrm{~g}$ replacement $=\bmod \left(\right.$ teacher,$\$\left\{n_{-}\right.$rooms $\left.\}\right)<1$ if year $==\$\{$ min_year $\}$

34 replace replacement $=$ replacement [_n-1] if year $>\$\{$ min_year $\}$

35 replace grade $=$ grade -1 if replace $==1$ \& year $>=1997$

36 replace school $=\bmod ($ school,$\$\{$ n_school $\})+1$ if grade $==$ $(\$\{$ min_grade $\}+1)$ \& year $>=1997$ \& replace $==1$

37 replace grade $=\$\{$ min_grade $\}+\$\left\{n \_g r a d e\right\}-1$ if grade $==(\$\{$ min_grade $\}+1)$

$\&$ year $>=1997 \&$ replace $==1$

38 replace grade $=$ grade +1 if replace $==1$ \& year $>=1997$ \& grade $<($ 
$\$\{$ min_grade $\}+1)$

39

40 * Generate true VA and class shocks

$41 \mathrm{~g}$ class_shock $=\operatorname{rnormal}(\Theta, \$\{$ sd_class_shock $\})$

$42 \mathrm{~g}$ va_true $=\operatorname{rnormal}(\odot, \$\{$ sd_va $\})$ if year $==\$\{$ min_year $\}$

43 replace va_true $=$ va_true [_n-1] if va_true==.

44

45 * Generate average lagged true VA and average lagged class shocks as

"double lags" of true

VA and class shocks

46 sort school year grade id

$47 \mathrm{~g}$ temp1 = va_true $\left[\_n-\$\left\{\mathrm{n} \_\right.\right.$rooms $\}$* $\left(\$\left\{\mathrm{n} \_\right.\right.$grade $\left.\left.\}+1\right)\right]$ if year $>$ $\$\{$ min_year $\} \&$ grade $>\$\{$ min_grade $\}$

$48 \mathrm{~g}$ temp2 $=$ temp1[_n $-\$\left\{\mathrm{n} \_\right.$rooms $\left.\} *\left(\$\left\{\mathrm{n} \_g r a d e\right\}+1\right)\right]$ if year $>\$\{$ min_year $\}$

$\&$ grade $>\$\{$ min_grade $\}$

$49 \mathrm{~g}$ temp3 = class_shock[_n - $\$\left\{\mathrm{n} \_\right.$rooms $\}$* $\left(\$\left\{\mathrm{n} \_\right.\right.$grade $\left.\left.\}+1\right)\right]$ if year $>$

$\$\{$ min_year $\} \&$ grade $>\$\{$ min_grade $\}$

$50 \mathrm{~g}$ temp4 = temp3[_n $-\$\left\{\mathrm{n} \_\right.$rooms $\left.\} *\left(\$\left\{\mathrm{n} \_g r a d e\right\}+1\right)\right]$ if year $>$ $\$\{$ min_year $\}$

$\&$ grade $>\$\{$ min_grade $\}$

51 by school year grade: egen 1_va_true = mean(temp1)

52 by school year grade: egen 12_va_true = mean(temp2)

53 by school year grade: egen 1_class_shock = mean (temp3)

54 by school year grade: egen 12_class_shock = mean(temp4)

55 drop temp*

56

57 * Generate school-by-year shocks

$58 \mathrm{~g}$ sy_shock $=\operatorname{rnormal}\left(0, \$\{\right.$ sd_sy_shock $\left.\} * \operatorname{sqrt}\left(1-\$\left\{r h o \_s y\right\} \wedge 2\right)\right)$ if $\bmod \left(\_n\right.$ ,$\$\left\{n_{-}\right.$rooms $\}$* $\$\left\{n_{-}\right.$grade $\left.\}\right)==1$

59 replace sy_shock $=$ sy_shock $/ \operatorname{sqrt}(1-\$\{$ rho_sy $\} \wedge 2)$ if year $==$

$\$\{$ min_year\} \& $\sim$ missing (sy_shock)

60 replace sy_shock = sy_shock[_n - 1] if missing (

sy_shock)

61 replace sy_shock $=$ sy_shock $+\$\{$ rho_sy $\}$ * sy_shock $\left[\_n-\$\{\right.$ n_rooms $\}$ *

$\$\left\{n \_\right.$grade $\left.\}\right]$if year >

$\$\{$ min_year $\}$

$62 \mathrm{~g}$ l_sy_shock = sy_shock[_n - $\$\left\{\mathrm{n} \_r o o m s\right\} * \$\left\{\mathrm{n} \_\right.$grade $\left.\}\right]$if year $>$

$\$\{$ min_year $\}$

63 g 12_sy_shock $=$ sy_shock[_n $\left.-2 * \$\left\{n \_r o o m s\right\} * \$\left\{n \_g r a d e\right\}\right]$ if year $>($

$\$\{$ min_year $\}+1)$

64

65 * Generate classroom average score, lagged, and twice-lagged scores

66 global sd_indv $=\operatorname{sqrt}\left(\left(\$\left\{v a r \_t o t\right\}-\$\left\{s d \_v a\right\} \wedge 2-\$\left\{s d \_s y \_s h o c k\right\} \wedge 2\right.\right.$ -

$\left.\$\{\text { sd_class_shock }\}^{\wedge} 2\right) /$

$\$\left\{\mathrm{n} \_\right.$class $\}$)

67 g 12_score $=$ (12_va_true + 12_sy_shock + 12_class_shock +

rnormal $\left.\left(\Theta, \$\left\{s d \_i n d v\right\}\right)\right)$

$68 \mathrm{~g}$ 1_score $=1$ l_va_true + 1_sy_shock +1 _class_shock $+\operatorname{rnormal}\left(\odot, \$\left\{s d \_i n d v\right\}\right)$

$69 \mathrm{~g}$ score $=$ va_true + sy_shock + class_shock $+\operatorname{rnormal}\left(\odot, \$\left\{s d \_i n d v\right\}\right)$

70

71 *Make dataset balanced panel

72 replace 1_score $=$. if 12_score $==$.

73 replace score $=$. if 1 _score $==$.

74

75 * Residualize scores using a single lag

76 sort teacher year

77 sum score

78 global tot_var $=r($ Var $)$ 
79 tsset teacher year

80 corr score l.score, c

81 global teach_var $=r\left(\operatorname{cov} \_12\right)$

82 global ind_var $=\left(\$\{\right.$ n_class $\left.\} /\left(\$\left\{n \_c l a s s\right\}-1\right)\right) *\left(\$\left\{v a r \_t o t\right\}\right.$ -

$\$\{$ tot_var\})

83 global class_var $=\$\{$ var_tot $\}-\$\{$ ind_var $\}-\$\left\{t e a c h \_v a r\right\}$

84

85 * Construct leave-two-out shrinkage and VA estimate

86 g temp $=$ inrange (year , 1996, 1997) \& missing(score)

87 by teacher: egen temp1 = sum(temp)

$88 \mathrm{~g}$ shrinkage $=\$\{$ teach_var $\} /(\$\{$ teach_var $\}+\$\{$ class_var $\} /$ temp1 + $\$\{$ ind_var $\} /\left(\$\left\{\mathrm{n} \_\right.\right.$class $\}$

* temp1))

$89 \mathrm{~g}$ temp2 = score if $\sim$ inrange $($ year $, 1996,1997) \& \sim$ missing $($ score $)$

90 by teacher: egen temp3 = mean (temp2)

$91 \mathrm{~g}$ va = temp3 * shrinkage if inrange $($ year, 1996, 1997)

92 drop temp*

93

94 * Construct Rothstein (2016) leave-three-out shrinkage and VA estimate

95 g temp $=$-inrange (year $, 1995,1997)$ \& -missing(score)

96 by teacher: egen temp1 = sum(temp)

97 g shrinkage_3out $=\$\{$ teach_var $\} /(\$\{$ teach_var $\}+\$\{$ class_var $\} /$ temp1 + $\$\{$ ind_var $\} /$ (

$\$\left\{\mathrm{n} \_\right.$class $\}$* temp 1$\left.)\right)$

$98 \mathrm{~g}$ temp2 = score if inrange (year , 1995, 1997) \& -missing(score)

99 by teacher: egen temp3 = mean (temp2)

$100 \mathrm{~g}$ va_3out $=$ temp3 * shrinkage_3out if inrange $($ year $, 1995,1997)$

101 drop temp*

102

103 * Construct prior shrinkage and VA estimate

$104 \mathrm{~g}$ temp $=$ (year < 1997) \& - missing (score)

105 bys teacher: egen temp1 = sum(temp)

$106 \mathrm{~g}$ shrinkage_prior $=\$\{$ teach_var $\} /(\$\{$ teach_var $\}+\$\{$ class_var $\} /$ temp1 + $\$\{$ ind_var $\} /$ (

$\$\{$ n_class $\}$ * temp 1$))$

$107 \mathrm{~g}$ temp2 = score if (year < 1997) \& -missing(score)

108 bys teacher: egen temp3 = mean (temp2)

109 drop temp*

110

111 save vam_simulation, replace

112

113 * Collapse data to school-grade-year level to implement quasi-

experimental analysis

114 keep if inrange (year, 1996, 1997)

115 collapse score 1_score va va_3out va_true, by(school grade year)

116 egen sy = group (school year)

117 egen $\mathrm{sg}=$ group (school grade)

118 tsset sg year

119 save vam_simulation_collapse, replace

120

121 * Results

122 log using lagged_score_simulation.smcl, replace

123 eststo clear

124 _eststo d_score: qui reg d.score d.va

125 _eststo d_score_sy: qui reg d.score d.va, a(sy)

126 _eststo d_score_3out: qui reg d.score d.va_3out

127 _eststo lag_d_score: qui reg d.l_score d.va 
128 _eststo lag_d_score_sy: qui reg d.1_score d.va, a(sy)

129 _eststo lag_d_score_3out: qui reg d.1_score d.va_3out

130 esttab_all, mtitles title("Quasi-Experimental Forecast Bias Estimates")

se not

$131 \log$ close

\section{Long-Term Effects of VA (Table 5)}

1 *This simulation shows that estimates of long-term impacts are downwardbiased in a multi-variable regression because VA is estimated with error and is correlated with $\mathrm{X}$

2

3 clear all

4 set seed 7817806

5 *Specify target: true effect of 1 unit increase in va_score on earnings

6 *Note that this is equivalent to effect of 1 unit increase in mu (not $\mathrm{m}=$ $\mathrm{mu} / \mathrm{sd}(\mathrm{mu}))$

7 global true_coeff $=100$

8

$9 * * * * * * * * * * * * * * * * * * * * * * * * * \operatorname{PART} 1$ 1 $9 * * * * * * * * * * * * * * * * * * * * *$

$10 * * * * * * * * * * * * * * * * * * * *$ Generate data $10 * * * * * * * * * * * * * * * * * * *$

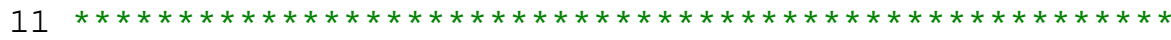

12

13 set obs 1000000

14 global n_class $=20$

15 global classes_per_teach $=10$

$16 \mathrm{~g}$ class $=\operatorname{ceil}\left(\right.$ $\left(\mathrm{n} / \$ \mathrm{n} \_\mathrm{class}\right)$

$17 \mathrm{~g}$ teacher $=\operatorname{ceil}\left(\_\mathrm{n} /\left(\$\left\{\mathrm{n} \_\right.\right.\right.$class $\} * \$\{$ classes_per_teach $\left.\left.\}\right)\right)$

18

$19{ }^{*}$ Generate test-score VA (mu_j)

20 bys teacher: $\mathrm{g}$ temp $=\ldots \mathrm{n}$

$21 \mathrm{~g}$ temp1 $=\operatorname{rnormal}(0,0.1)$ if temp $==1$

22 bys teacher: egen va_score $=$ mean (temp 1$)$

23

$24{ }^{*}$ Generate pure earnings component of VA

$25 \mathrm{~g}$ temp2 $=\operatorname{rnormal}(\odot, 0.1)$ if temp $==1$

26 bys teacher: egen va_earn = mean $($ temp2)

27 drop temp*

28

$29{ }^{*}$ Generate total earnings VA (tau_j)

$30 \mathrm{~g}$ va_comb = va_score + va_earn

31

$32{ }^{*}$ Generate covariate $X$ correlated with teacher's total earnings VA

33 global rho $=0.33$

$34 \mathrm{~g} x=\left(\$\{r h o\}^{*} v a \_c o m b+(1-\$\{r h o\}){ }^{*} \operatorname{rnormal}(\odot, 0.1)\right) / \operatorname{sqrt}(\$\{r h o\} \wedge 2+(1-$ $\left.\$\{r h o\})^{\wedge} 2\right)$

35

$36{ }^{*}$ Generate scores and earnings

37 *Note that only va_score affects test scores, while both va_score and

va_earn affect earnings

$38 \mathrm{~g}$ score $=$ va_score $+x+\operatorname{rnormal}\left(\odot, \operatorname{sqrt}\left(1-.1^{\wedge} 2\right)\right)$

$39 \mathrm{~g}$ earn $=\$\{\text { true_coeff }\}^{*}$ va_comb $+10^{*} \mathrm{x}+\operatorname{rnormal}(0,10)$

40

$41 * * * * * * * * * * * * * * * * * * * * * * * \mathrm{PART} 2$.

$42 * *$ Estimate VA using within-teacher residualization** 


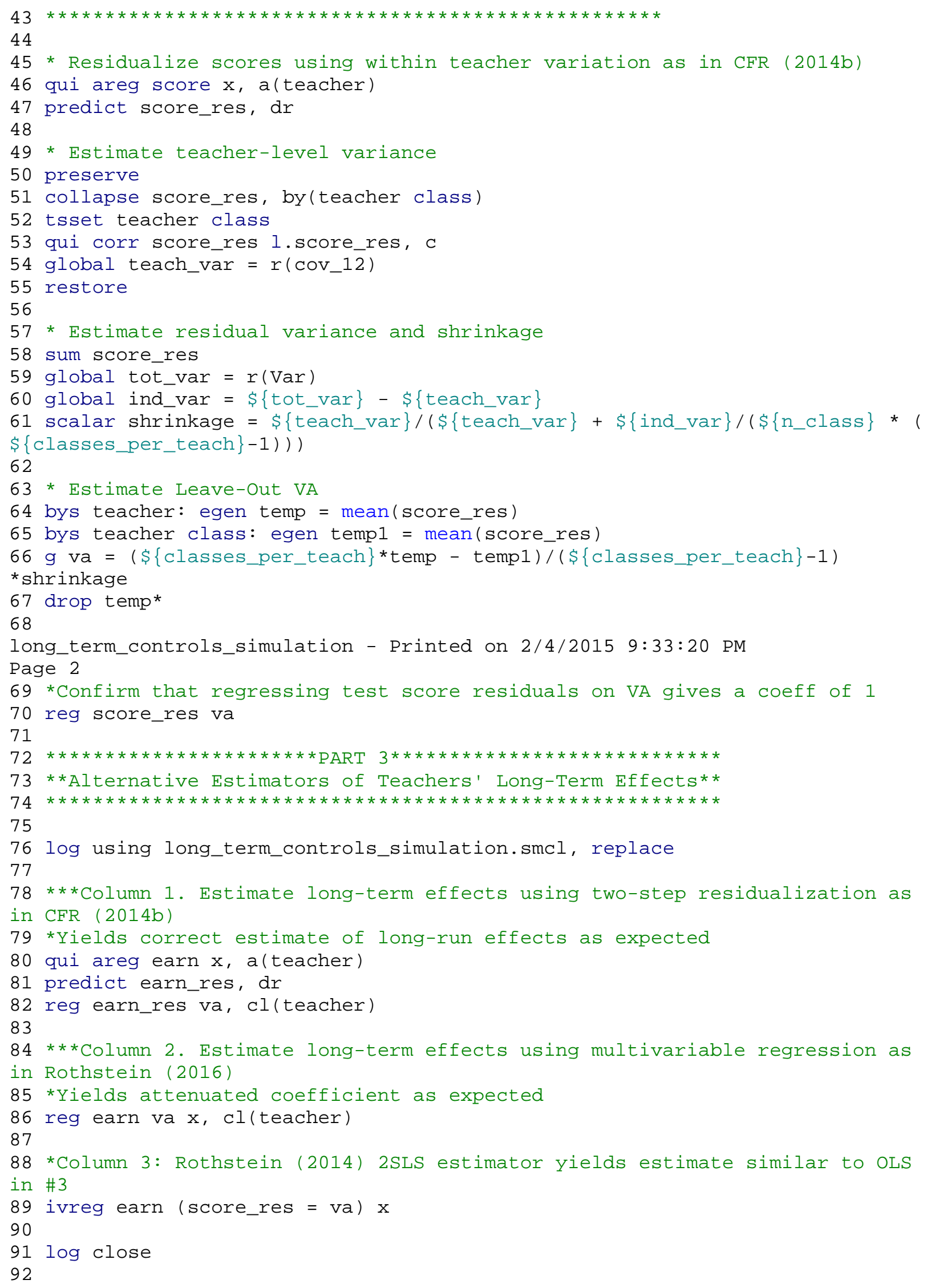

